\title{
Influence of Different Meltable Binders on the Solid- State Behaviour and Dissolution Profiles of Solid Lipid Extrudates Processed Via Continuous Hot Melt Granulation
}

Santosh Gejage ${ }^{1}$, Jaywant Pawar ${ }^{1}$, Purnima Amin ${ }^{1 *}$, and Mohammed Maniruzzaman $^{2 *}$

1. Department of Pharmaceutical Sciences and Technology, Institute of Chemical Technology, Elite Status, Nathalal Parekh Marg, Matunga, Mumbai 400019, India.

2. Department of Pharmacy (Chemistry), School of Life Sciences, University of Sussex, Falmer, Brighton BN1 9QT, United Kingdom

*Corresponding author:

Dr Mohammed Maniruzzaman $\quad$ (M.Maniruzzaman@sussex.ac.uk, or M.Maniruzzaman12@gmail.com) and Prof. Purnima, D. Amin (email purnima.amin@yahoo.co.in) 


\begin{abstract}
:
Mere similar grades of same excipients manufactured by two different manufacturers often differ significantly that they even have an impact on the variations in final product or dosage form. Solid lipids are one of the most available options as matrix former in sustained drug delivery. Due to their chemical and physical complexity, lipids may exhibit a complex behaviour (i.e. melting crystallization and polymorphism). The aim of this study was to evaluate the physical and chemical properties of two Glyceryl Monostearate (GMS) lipids (Geleol from Gattefosse and Capmul GMS 50K from Abitec Corp. USA) and how these properties may affect during melt granulation process for sustained release applications. Thermal processing was applied on GMS samples to understand the polymorphic nature and suitability as meltable binders. Niacin was used as model drug. A thorough evaluation of GMS samples and sustained release tablets was undertaken using analytical techniques such as differential scanning calorimetry (DSC), X-ray diffraction (XRD). Moreover, melt viscosity study assisted to apprehend the behaviour of GMS samples in hot melt extrusion processing. Surface morphology of the drug and extruded granules examined via SEM and AFM revealed high level of surface interaction and dense structure of drug inside lipid matrix. The DSC and XRD study confirmed that Geleol could not withstand the heat treatment applied during thecontinuous melt granulation processing. The shifting of stable $\beta$ form to an unstable $\alpha$ form in Geleol was detected. In the contrary case, Capmul GMS 50K was able to withstand the heat treatment supported by the applied analytical techniques. Nonetheless, both GMS samples perform differently in final dosage form. This change in stable $\beta$ form to an unstable $\alpha$ form affected dissolution profiles at 3 months storage in accelerated condition. This study helped to interpret the complex solid-state behaviour of solid lipid extrudates with different compositions, in order to the simply and outline a suitable formulation strategy for the development of lipid-based oral dosage forms.
\end{abstract}



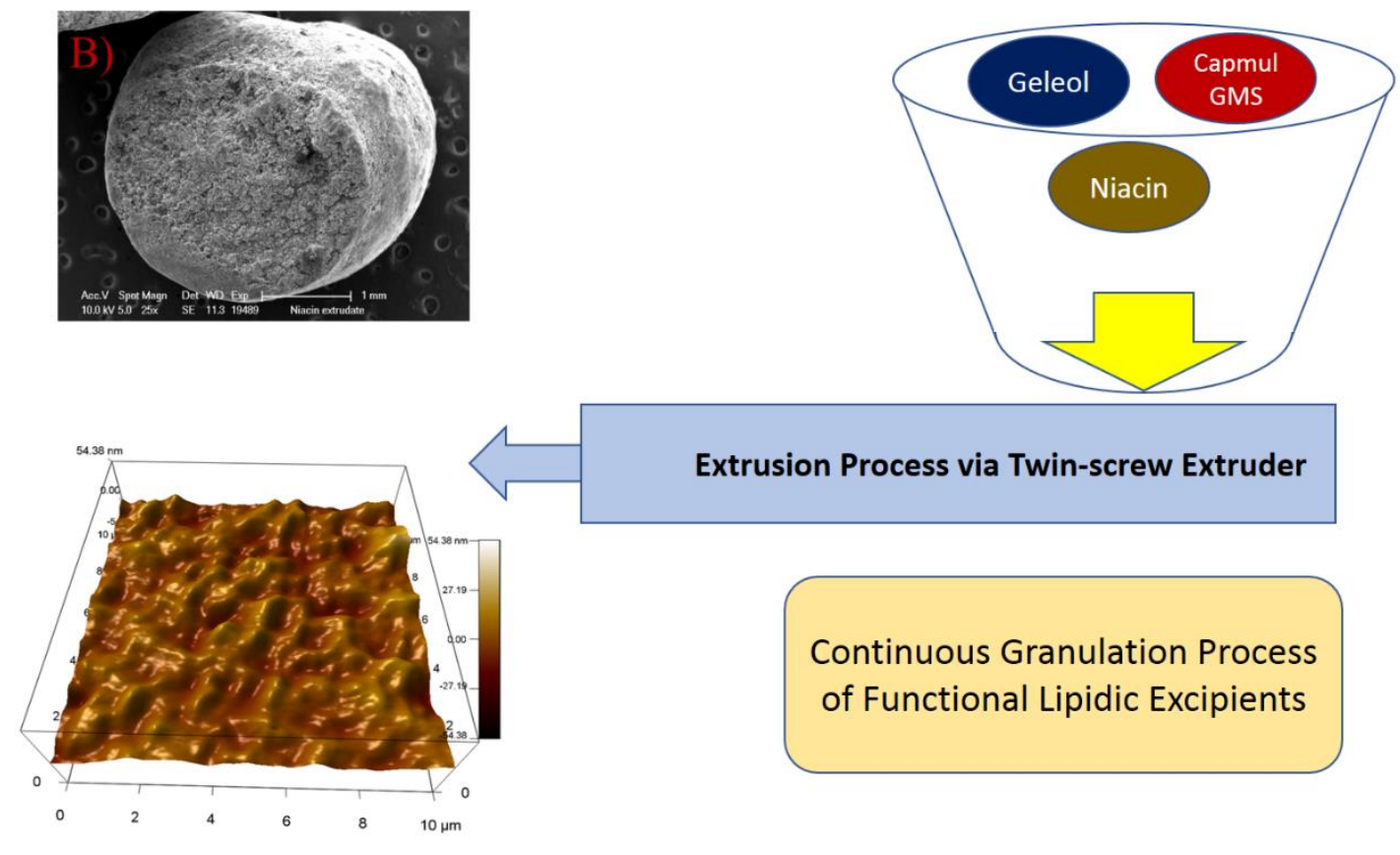

Graphical Abstract

\section{Keywords:}

Niacin, Glyceryl Monostearate, Polymorphism, Melt viscosity, hot melt extrusion, Melt granulations, Sustained release.

\section{Introduction:}

Quality control tests must be performed on excipients or powders to ensure certain attributes of the excipients or powder to fall within a predefined range. These attributes include but are not limited to chemical composition, particle size, color, moisture and flow properties. In this context, this study examines a thorough investigation of two similar lipids manufactured by different manufcatures which would help the formulation scientist to screen the excipients before or at the outset of the actual development of target finished products (e.g. tablets). Formulation scientist should not be biased when thethe selection of the most appropriate excipients is concerened. Mere similar grades of same excipients manufactured by two different companies will not be always same and they do have an impact on the variations in finished products or dosage forms. Different excipients are generally used to manufacture tablets. In order to develop sustained release tablets different class of materials such as cellulose derivatives [Hydroxypropyl cellulose 
(HPC), Hydroxy propyl methyl cellulose (HPMC), Ethyl cellulose (EC)], Carbomers, acrylates (Evonik polymers) and lipids (waxy and having HLB <5) are generally used to as a carrier matrix which often retard the drug release. Pharmaceutical grade polymers are widely used in twin screw hot melt extrusion (HME) processing. They enable continuous manufacturing of solid solutions and thus extended release dosage forms. However, high processing temperatures and the need for plasticizers sometime force the formulators to explore alternative extrudable materials, including solid lipid excipients [1,2]. The underlying reason is being that the solid-lipid matrices can be processed via HME at significantly lower temperatures without the need for plasticizers. Moreover, lipid excipients have a wide range of applications in pharmaceutical industry. Solid glyceryl stearates are commonly used as matrix formers in sustained release tablets and capsules [3]. The melt granulation, most broadly defined as a powder agglomeration with the help of a meltable binder, is applied in numerous pharmaceutical products for a variety of reasons, e.g., to facilitate compression, improve flow, uniformity and to enhance or control dissolution profiles of the drugs used [4].

Commonly, reported binders such as polyethylene glycol, HPC, HPMC, PEO and HPMC in combination with lipid binders is used in melt granulation [5-7]. It has been reported in the literature that polymeric binders are not preferable when granulation process is developed for modified or controlled release applications due to solubility in ethanol and susceptibility to dose dumping in hydroalcoholic media [5-7]. To overcome the problem of polymeric meltable binders solid lipids are one ofmost viableoptions. A numerous pharmaceutical grade lipid excipients have been commercialized by different companies over the past several decades for the development of drug delivery systems [8,9]. The continuous processing of granules, suitable for continuous blending/compression and for sustained release from a lipid matrix (10)such as glyceryl monostearate (GMS), glyceryl dibehenate, glyceryl tripalmitate, glyceryl tristearate etc has been demonstrated [10-12]. The physicochemical properties of complex naturally occurring lipid compounds were optimized by modifying their chemical composition (e.g. establishing defined mixtures of esterified fatty acids) and by processing fatty acids with various functional groups such as glycerol, polyethylene glycol, polyglycerol or propylene glycol. By doing so, the melting point (range) and hydrophilic lipophilic balance (HLB) value were adjusted in relation to a given application. Solid lipid excipients with high melting point (long acid chain length) and low HLB value (less polar alcohol) are suitable to retain water soluble drug molecules within the dosage 
form [13]. Due to their chemical and physical complexity, meltable lipid binders may exhibit a complex behaviour (i.e. melting and crystallization, physical modifications on storage) [14, 15] which has not been thoroughly investigated before.

Niacin is used as a model drug which is an anti-hyperlipidemic agent currently marketed as an oral sustained release dosage form. Niacin is a white, crystalline powder, very soluble in water. Glyceryl Monostearate is a lipid excipient suitable for the controlled release of water soluble drugs. GMS is a mixture of monoacylglycerols mainly monostearoylglycerol together with variable quantities of di and triacylglycerols that has a melting range between $54^{\circ} \mathrm{C}$ and $65^{\circ} \mathrm{C}$. GMS is manufactured by different manufacturers worldwide, each has a different process and has a different composition of Monoglycerides, diesters and triesters contents and also different polymorphic forms. And this composition may have different effects on the final formulation or during stability studies.

Therefore, the aim of this study is to evaluate the physical and chemical proerties of two GMS samples manufactured by two different companies. This evaluation is particularly useful for the development of controlled-release formulations as the melting and rheological characteristics of fatty acid binders are most critical parameters to consider, especially when a high amount of lipidic binder mixtures are used. Another objective of this study is to examine a comparative evaluation of GMS as a meltable binder from two different sourcesduring a melt granulation process for sustained release applications.

\section{Materials and Methods:}

\subsection{Materials:}

Glyceryl Monostearate(GMS) with brand name Geleol ${ }^{\circledR}$ was received from Gattefossé India Pvt. Ltd and Capmul GMS-50K ${ }^{\circledR}$ from Abitec Corp. Mumbai. GMS occurs as fine, white free flowing powders. Niacin IP was purchased from Ishita Drugs \& Industries Ahmedabad, India. Microcrystalline cellulose (Avicel ${ }^{\circledR}$ PH-102) and Hydroxy Propyl cellulose (Aqualon ${ }^{\circledR}$ LF grade) were received as free gift from Signet Chemical Corporation Mumbai, India. Magnesium stearate, Aerosil $200^{\circledR}$, and Talc were used for the tableting study and procured from Sigma Aldrich. All the other reagents and solvents used were of analytical grade. 


\subsection{Samples Preparation:}

As described in previous work (J. Hamdani et. al) [16] three samples were prepared for the physical evaluation of GMS.

- Freshly solidified (F) samples: Samples were previously melted by heating at a temperature at least $10^{\circ} \mathrm{C}$ higher than their melting point, solidified at ambient temperature, and stored for $10 \mathrm{hr}$ at room temperature in order to see effect melt processing on lipids.

- Aged (A) samples: Samples were stored for 48 hours at $50^{\circ} \mathrm{C}$, in order to detect any physical ageing effect (structural or polymorphic changes) associated with lipids bases.

- Untreated (U) samples: Samples without any special treatment (as received).

\subsection{Differential Scanning Calorimetry:}

The melting behavior of the lipophilic binders was evaluated by a Perkin Elmer pyris-6 (USA) which has an inbuilt computerized data station for thermal analysis. (Perkin-Elmer Corp., CT, USA). Samples of about $5 \mathrm{mg}$ were sealed in aluminum pans and scanned between 0 and $150{ }^{0} \mathrm{C}$, at a heating rate of $10^{0} \mathrm{C} / \mathrm{min}$ under nitrogen flow $(20 \mathrm{ml} / \mathrm{min})$ using empty sealed pan as reference.

\section{$2.4 X$-ray Diffraction}

Crystallinity of the raw materials, hot-melt extrudates and tablets samples was determined using Lab XRD 6100 Shimadzu x-ray Diffractometer in Theta-Theta mode, Cu anode at $40 \mathrm{kV}$ and $30 \mathrm{~mA}$ current. The samples were pulverized in a ball mill chamber with a frequency of 25 $\mathrm{s}-1$ for $30 \mathrm{~s}$. The powdered sample was placed on a glass top loading holder with a depression of $0.2 \mathrm{~mm}$ and then gently consolidated. All samples were scanned in a continuous mode within the angular range $5-60^{\circ} 2 \theta$ scale with a scan speed of $2.0^{\circ}$ per min and sample width of $0.03^{\circ}$.

\subsection{Scanning Electron Mircoscopy}

The shape and surface morphology of the niacin powder and niacin loaded extrudates were examined using XL 30 Model JEOL 6800 scanning electron microscope made in Japan during analysis. Double-sided carbon tape was affixed on aluminium stubs over which powder sample of 
niacin was sprinkled and prepared extrudates was attached carefully. The radiation of platinum plasma beam using JFC-1600 auto fine coater was targeted on aluminium stubs for its coating to make layer of $2 \mathrm{~nm}$ thick- ness above the sprinkled powder for $25 \mathrm{~min}$. Then, those samples were observed for morphological characterization using a gaseous secondary electron detector (working pres- sure: 0.8 Torr, acceleration voltage: $10 \mathrm{e} 30.00 \mathrm{kV}$ )

\subsection{Atomic Force Microscopy (AFM) Characterization}

JXA-8530F Hyper Probe Electron Probe Microanalyzer instrument by JEOL was employed for AFM analyses. Freshly fractured extrudates on microscopic glass slides were mounted on the micrometre positioning stage of a Dimension Icon AFM with accelerating voltage of 1-30 kV. Probecurrent rangewas kept between 10 pAand 200 pAand back scattered electron images were obtained [17].

\subsection{Melt Viscosity measurements}

The rheological properties of two GMS samples were determined on the solid material, at different temperatures close to their melting point, using a Viscolead One model viscometer (FungiLab Spain,) spindle L3 at 50 rpm speed. 8-10 gms sample was filled in small sample adaptor which was then attached to the main viscometer. Small sample adaptor was immersed in a digital water bath to maintain the temperature.

\subsection{Continuous melt granulation processing of Niacin using GMS as matrix former}

\subsubsection{Continuous melt Granulation}

Niacin as received contained a substantial fraction of particles greater than $250 \mu \mathrm{m}$. prior to granulation, niacin was passed through sieve no 140 (106 $\mu \mathrm{m}$ threshhold). The fraction less than $100 \mu \mathrm{m}$ was collected for further processing and analysis. Melt granulation of niacin with Gelol and Capmul GMS 50K were performed by twin screw extruder instrument (ACG Hot Melt Extruder). Niacin was mixed with GMS and HPC LF at drug/lipid mass ratios of using a V-blender (Wintech Pharmachem Equipment Pvt. Ltd Mumbai) for 5 min. The prepared physical mixtures (PMs) were extruded using a co-rotating single-screw extruder at a screw speed of $50 \mathrm{rpm}$ in order to optimize the formulation and processing parameters prior to scaling up to a twin-screw extruder. For all experiments, the extruder was operated without a die or transfer plate. Optimized batches 
were then processed using twin screw extruder instrument (ACG Hot Melt Extruder). The melt extrudate was then milled using a grinder, passed through a $200 \mu \mathrm{m}$ sieve and packed in HDPE airlock containers for furtheranalyses. HME processing speed, extrusion temperature (Tg mix) and feeding rate were found to be controllable parameters in the process that could affect chemical stability of formulation. All extrusions were performed in triplicate to measure the processing torque and mean residence time (MRT). Melt granulation was processed through the extruder with all temperature control points set at $75^{\circ} \mathrm{C}$, which is above the melting temperature range of GMS.

\subsubsection{Blending and compression}

Niacin granules from above step (2.6.1) and other excipients intended to act as a pore former were blended for $15 \mathrm{~min}$ in a V-Shell blender (Wintech Pharmachem Equipment Pvt. Ltd Mumbai) operating at $25 \mathrm{rpm}$ followed by an additional 5 min of blending with the tableting lubricant Aerosil ${ }^{\circledR}$ 200, Talc and magnesium stearate. The batch size for the tablet blends was 100 g. Individual portions of the blends were weighed and compressed into 19.1 X $8.1 \mathrm{~mm}$ plain caplet shape tablets at $20 \mathrm{kN}$ compression force using a tablet press, (Manual Tablet Compaction Machine, model CMD-4, Cadmach Machinery Co. Pvt. Ltd India). The composition of tablets is shown in Table 1.

Table 1: Selected formulations of Niacin sustained-release tablets

\begin{tabular}{lcccccc}
\hline \multicolumn{1}{c}{ Ingredients } & \multicolumn{5}{c}{ Formulation Batches } \\
\cline { 2 - 7 } & G1 & G2 & G3 & A1 & A2 & A3 \\
& 375 & 375 & 375 & 375 & 375 & 375 \\
Niacin & 160.71 & 250 & 375 & 160.71 & 250 & 375 \\
Lipid & 139.89 & 48.8 & 25.4 & 139.89 & 48.8 & 25.4 \\
MCC & 36.5 & 36.5 & 20 & 36.5 & 36.5 & 40 \\
HPC LF (Aqualon) & & & & & & \\
Talc & 7.3 & 7.3 & 8.2 & 7.3 & 7.3 & 8.2 \\
Magnesium Stearate & 7.3 & 7.3 & 8.2 & 7.3 & 7.3 & 8.2 \\
& & & & & & \\
Aerosil 200 & & & & & & \\
Tablet weight in mg & 7.3 & 7.3 & 8.2 & 7.3 & 7.3 & 8.2 \\
& 734 & 732 & 820 & 734 & 732 & 820 \\
\hline
\end{tabular}




\subsection{In vitro dissolution study}

An in-vitro drug release of niacinamide tablets was studied byusing dissolution apparatus I-Basket (Electrolab Model TDT 08, India) as per OGD media USFDA datasheet. $900 \mathrm{~mL}$ of purified water as the dissolution medium was placed in the dissolution vessels, and the temperature was maintained at $37 \pm 0.5{ }^{\circ} \mathrm{C}$. The rotation speed of paddle was $100 \mathrm{rpm}$. At predetermined time intervals $(1,3,6,9,12$ and 20 hour), $10 \mathrm{~mL}$ of dissolution medium was removed for determining a drug concentration and was replced by equal amount of fresh medium. The amount of niacin released in the dissolution medium was measured using a UV spectrophotometer at wavelength of $262 \mathrm{~nm}$. The dissolution study was performed using 6 tablets in triplicate $(n=3)$.

\subsection{Stability Studies:}

Stability studies were conducted on manufcatured tablets at $40^{\circ} \mathrm{C}$ and $75 \%$ relative humidity conditions. Thirty tablets were distributed, under ambient conditions, into $50 \mathrm{cc}$ HDPE bottles and induction sealed for $4 \mathrm{~s}$ using an induction sealing machine (Eltech, H500 Induction sealer). Packaged tablets were placed in a laboratory stability chamber set for and controlled to $40^{\circ} \mathrm{C}$ and $75 \% \mathrm{RH}$. A sealed unopened bottle was collected and evaluated initially, at 1 month and 3 months.

\subsection{Results and discussion}

\subsection{Pharmaceutical status of GMS and Profile of GMS}

Pharmaceutical industry knows various applications of glyceryl monostearate as nonionic emulsifiers, stabilizers, emollients, and plasticizers in a variety of food, pharmaceutical, and cosmetic applications. GMS can be utilized via a novel fluidized hot-melt granulation technique for the production of granules and tablets. Due to its low HLB and hydrophobic nature, it is used to form sustained-release matrices for solid dosage forms such as pellets or tablets. While the names glyceryl monostearate and mono- and diglycerides are used for a variety of esters of longchain fatty acids, the esters fall into two distinct grades which are available in market [18].

40-55 percent monoglycerides: The PhEur 6.0 defines glyceryl monostearate 40-55 as a mixture of monoacylglycerols, mostly monostearoylglycerol, composed of quantities of di- and 
triacylglycerols. It holds 40-55\% of monoacylglycerols, 30-45\% of diacylglycerols, and 5-15\% of triacylglycerols. This PhEur grade corresponds to mono- and di-glycerides USP- NF, which has analogous specifications (not less than $40 \%$ monoglycerides).

90 percent monoglycerides: The USP32-NF27 defines glyceryl monostearate as comprising of not less than $90 \%$ of monoglycerides of saturated fatty acids, primarily glyceryl monostearate $\left(\mathrm{C}_{21} \mathrm{H}_{42} \mathrm{O}_{4}\right)$ and glyceryl monopalmitate $\left(\mathrm{C}_{19} \mathrm{H}_{38} \mathrm{O}_{4}\right)$. The commercial products are blends of variable amounts of glyceryl monostearate and glyceryl monopalmitate. Glyceryl monostearate is a white to cream-colored, wax-like solid in the form of beads, flakes, or powder. For our research study, we have selected GMS of 40-55 percent monoglycerides composition. For the in-depth study of two GMS samples viz, Geleol ${ }^{\circledR}$ were received from Gattefossé India Pvt. Ltd and Capmul GMS-50K ${ }^{\circledR}$ received from Abitec Corp. Mumbai. Comparison of major COA parameters are reported in Table 2 .

Table 2: Major COA parameters of two GMS samples

\begin{tabular}{llllc}
$\begin{array}{l}\text { Sr. } \\
\text { No }\end{array}$ & Characteristic & Specification & $\begin{array}{l}\text { Capmul } \\
\text { GMS-50K }\end{array}$ & Geleol \\
\hline $\mathbf{1}$ & Appearance & Waxy Solid & Conforms & Conforms \\
$\mathbf{2}$ & Odour & Faint & Conforms & Conforms \\
$\mathbf{3}$ & Melting Point & 54.0 to $64^{0} \mathrm{C}$ & 58.3 & 57.2 \\
$\mathbf{4}$ & Total Monoglycerides & 42.3 to $51.7 \%$ & 53 & 51.4 \\
& Content & 30.0 to $45.0 \%$ & 38.1 & 40.6 \\
$\mathbf{5}$ & Total Diesters content & 5.0 to $15.0 \%$ & 6.8 & 7.5 \\
$\mathbf{6}$ & Total Triesters content & 40.0 to $60.0 \%$ & 53.2 & 51.2 \\
$\mathbf{7}$ & Stearic Acid & $>=90 \%$ & 94.1 & 98.4 \\
$\mathbf{8}$ & Sum of Palmitic acid and & & & \\
\hline
\end{tabular}

From the Table 2, it is clear that both the selected products comply the pharmacopeial tests. The ranges given for compositions of fatty acids and melting points are also comparable.

\subsection{Polymorphism in Glyceryl Monostearate via solid state analysis}

Laine et. al 1988 reported the crystallization rate depends on the size of molecules i.e on the number of carbon atoms in the fatty acid chains. Longer the fatty acid chain, longer would be the 
crystallization time [19]. Laine et. al 1988 have evaluated the layered structure of tricaprin, trimyristin, and tristearin. Opposite to this Hamdani et. al 2002 have reported that the compritol 888 ATO which is a behenate having the C22 structure reported the substantially higher stability of this lipid [16]. In our case the stearate which is having C21 structure which takes less time as the layered structure disappears fastly. Finally, in order to identify the difference between two GMS samples during processing and at long-term storage conditions the freshly solidified samples (melting $10^{\circ} \mathrm{C}$ above their melting point and cooling at ambient temperature) and aged samples were evaluated with the help of DSC and XRD.

The objective of the solid state analysis was to evaluate the effect of physical and chemical characterization of GMS samples on the performance of materials. Due to their chemical and physical complexity, the meltable lipid binders may exhibit a complex behavior (i.e. melting and crystallization, physical modifications on storage). Proper selection of the polymorphic form of GMS in a formulation is very important. Two basic polymorphs of GMS are reported in literature -an unstable alpha plus a stable beta form. The variability in the composition of GMS was studied in detailed, firstly by Raymond. et. al., [20] They have reported the diffraction data and suggested that GMS was undergoing a slow, but continuous, change in its crystalline state. T. Yajima also characterized the polymorphic forms and effect of heat on the polymorphic content of GMS by using DSC and X-ray diffractions [21].

It is observed that the endotherms of three different samples are not in accordance with the specifications values. This was unexpected because the specifications termed a melt in between 54.0 to $64^{\circ} \mathrm{C}$ by a sealed-tube method. In the investigation of DSC endotherms, the values are in the range of 64 to $70^{\circ} \mathrm{C}$. The fact that the reported melting point for pure GMS is $81.5^{\circ} \mathrm{C}$ which is of high-melting component i.e purer material. Raymond et. al. 1968 concluded that changes in the polymorphic form in GMS results in a specific problem, i.e. the stability of a particular formulation that leads to product breakdown (19). The groupdemonstrated how emulsions made by GMS were unstable due to polymorphic changes during the stability studies. The polymorphic transitions of lipid excipients is critical for drug incorporation and stability, particularly for the nanoparticulate drug delivery systems based on GMS. Polymorphic stability of GMS and its polymorphic transition are very important factors to be considered when designing lipid based formulations [22, 23]. 
It was our concern that whether the GMS could behave in the same manner in melt granulation process? To find the answer to this question we have conducted several analytical experiments such as DSC and XRD with different heat treatments to find out the polymorphic nature of two different GMS samples. The presence broad melting endotherm was detected in untreated Geleol sample which is having a peak at $69.25^{\circ} \mathrm{C}$ that suggests all the material is in a melted state. However, the small shift in melting endotherm was observed during storage at $50^{\circ} \mathrm{C}$ for 48 hours with an increase in the height and sharpness of melting endotherm. This was in agreement with previous observations by Jingying et al. In the similar case Hamdani et al., 2003 has reported, the findings about precirol and compritol 888 ATO when processed with heat treatment. With the help of DSC and HSM methods, they have shown the presence of polymorphism for Precirol. Moreover, both untreated and fresh solidified Precirol and Compritol samples present to a degree amorphous layered structure which gradually crystallize in time. They reported that the rate of crystallization was more rapid for Precirol and highly reliant on the ageing conditions (storage temperature). Similar observations were reported elsewhere that enthalpy change (E) of GMS was dependent on the temperature profile $[16,21,23]$. When the temperature is below $50^{\circ} \mathrm{C}$, enthalpy increases, and when the temperature is aove $50^{\circ} \mathrm{C}$ enthalpy decreases. With supporting transformation rate equations they have recommended $50^{\circ} \mathrm{C}$ will be the optimum processing temperature for the transformation of GMS from $\alpha$ form to $\beta$ form. The more stable $\beta$ forms of GMS is a crystalized lattice with high packing density, which have left limited space for drug molecules between the lipid lattice structure. In the melt granulation process drug is granulated with the melted lipid binder under pressure. High pressure treatment can affect the crystal morphology, crystal size distribution, polymorphic properties [24]. Since the drug is covered with lipid binder, the physical and chemical stability of meltable binder plays an important role in final stability of the dosage form.

To understand the polymorphic structure after heat treatment that resembles the melt extrusion step. We have melted both lipids above $10^{\circ} \mathrm{C}$ to the melting points and cooled to room temperature and kept for 24 hours prior to the analytical study. When DSC endotherms were recorded on these samples Geleol has shown shift in melting endotherm also the sharpness of endotherm was also notable. While the untreated Capmul GMS 50K samples show similar broad endotherm (Fig 1(b)) but after heat treatment the sharpness of endotherm is distinguished.

Practically there is less difference in the peak temperature in the two Capmul sample. In 
contrast, the Capmul GMS $50 \mathrm{~K}$ samples on storing to $50^{\circ} \mathrm{C}$ started shifting the endotherms on higher side. The probable reason that the unstable $\alpha$ form started shifting towards stable $\beta$ form. The DSC endotherms showed in Fig. 1(c) permit to confirm the complexity of ageing modifications observed with two GMS samples. And these DSC endotherms are well linked with X-ray diffraction patterns of GMS samples. The x-ray diffraction patterns obtained from two untreated GMS samples permit to observe and that there is no superimposition between Geleol and Capmul GMS $50 \mathrm{~K}$ sample and are depicted in Fig. 2 (a-c). The X-ray diffraction pattern of fresh Geleol shows three major peaks at a $2 \theta$ angle of $19.70^{\circ}, 22.94^{0}$ and $23.26^{\circ}$. Similar nature of diffraction patterns was also observed for Capmul GMS 50K (Fig. 2(b)). The diffraction patterns of Capmul GMS 50K show three peaks at $2 \theta$ angles of about $19.53^{\circ}, 22.58^{\circ}$ and $23.18^{0}$. Aged sample (48 hours at $50{ }^{\circ} \mathrm{C}$ ) of two GMS sources identified and showed similar X-ray diffraction pattern. Moreover, quite different diffraction patterns of two GMS samples were observed in case of freshly solidified samples. Geleol, with individual peaks, were merged in a single broad peak having one intense peak at a $2 \theta$ angle of $22.78^{\circ}$. This single broad peak confirmed the shifting towards an unstable $\alpha$ form. Contrary to this the in capmul GMS 50K freshly solidified samples crystal structure was well stable as compared to untreated samples. This confirms the capmul GMS can withstand the heat treatment. There is no change in its $\beta$ form after heat treatment. 


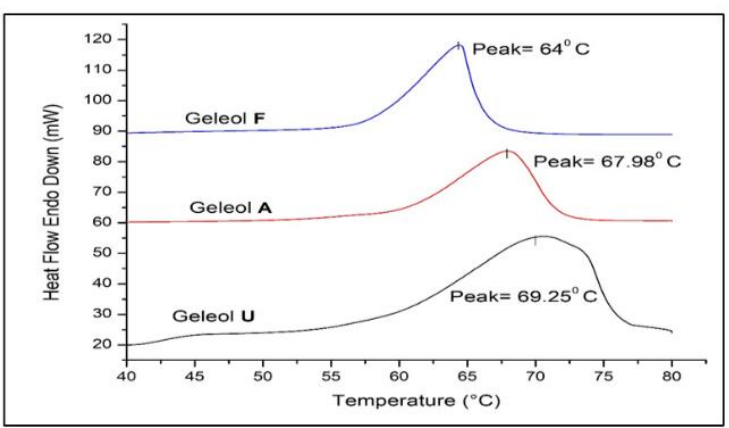

(a)

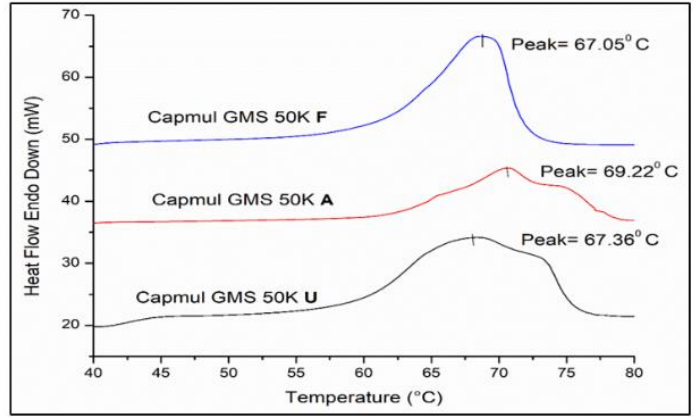

(b)

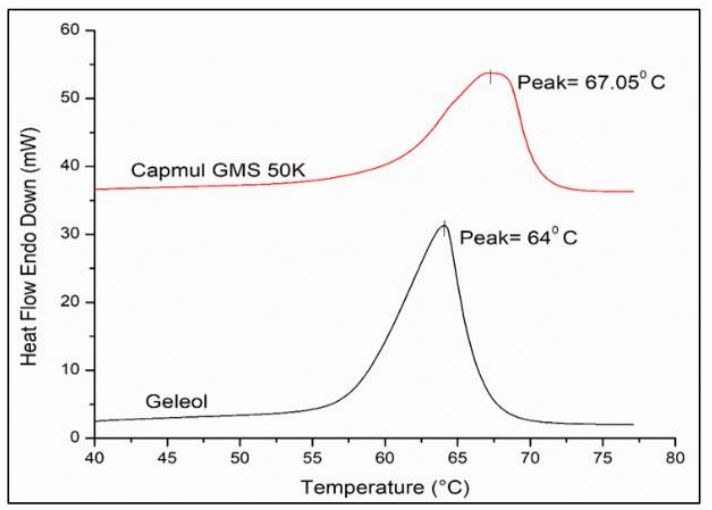

(c)

Figure 1: (a) DSC heating curves of Geleol obtained from untreated (U), Freshly solidified (F) and aged (A) (stored at 48 hours at $50{ }^{\circ} \mathrm{C}$ ) samples; (b) DSC heating curves of Capmul GMS 50K obtained from untreated (U), Freshly solidified (F) and aged (A) (stored at 48 hours at $50{ }^{0} \mathrm{C}$ ) samples; (c) DSC heating curves of Capmul GMS 50K and Geleol of Freshly solidified (F) samples.

These observations informs not only the importance of cooling and heating rate during processing on the samples physical state but also the instability of some polymorphic forms of glyceride bases after crystallization. Indeed, during these investigations, the appearance of low melting range endotherms of GMS was only detected when the heating-cooling programs were applied on the samples in DSC instrument, i.e. when DSC thermograms were performed on the freshly solidified samples immediately after crystallization. This is probably the reason why appearance of polymorphs of geleol was detected by the X-ray diffraction. In contrast, this phenomenon was not observed for Capmul GMS 50K, for which DSC curves obtained are characterized by a more limited and sharp melting range, showing only one important melting endotherm at approximately $67.05^{\circ} \mathrm{C}$. Moreover, a careful evaluation of the X-ray diffraction peaks permits to observe that both Geleol and Capmul GMS 50K samples show different 
diffractograms in comparison with the freshly solidified samples. This confirms they may affect performance. This will be evaluated by making tablets and evaluating under stress conditions.

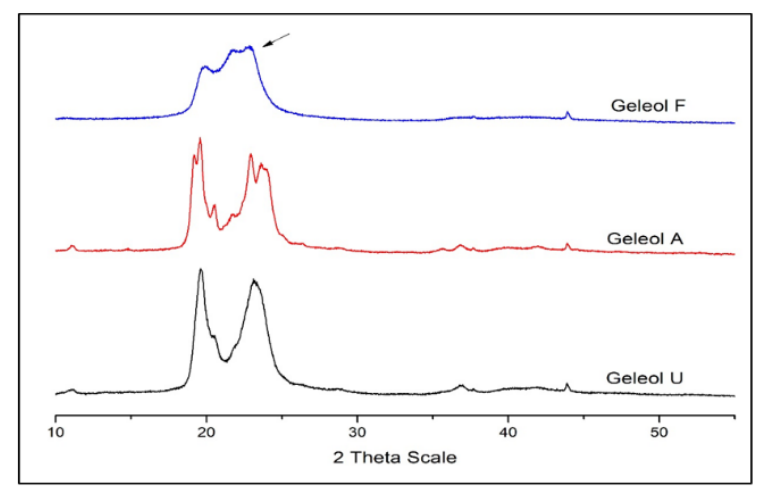

(a)

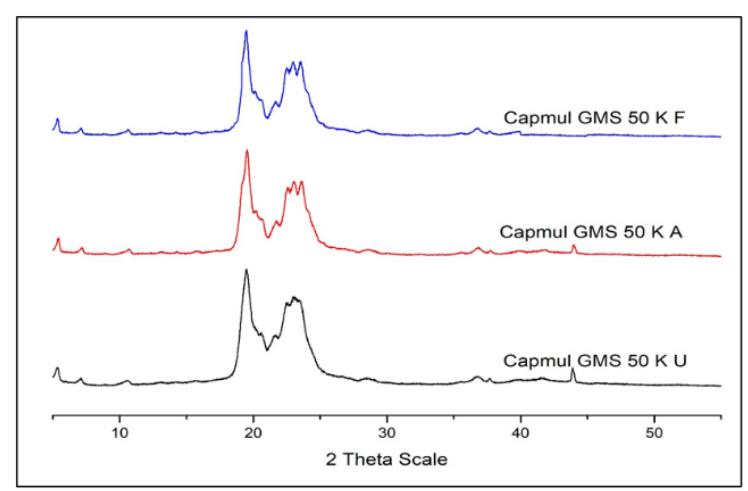

(b)

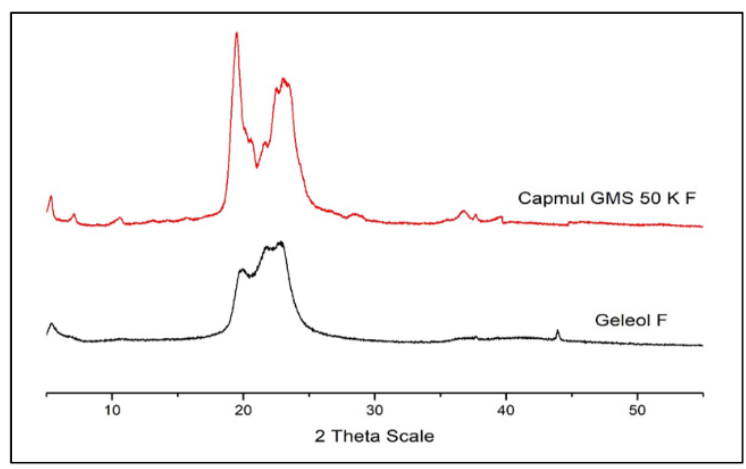

(c)

Figure 2: (a) X-ray Diffractograms of Geleol obtained from untreated (U), Freshly solidified (F) and aged (A) (Stored at 48 hours at 500 C) samples; (b) X-ray Diffractograms of Capmul GMS $50 \mathrm{~K}$ obtained from untreated (U), Freshly solidified (F) and aged (A) (stored at 48 hours at 500 C) samples; (c) X-Ray Diffractograms of Capmul GMS 50K and Geleol of Freshly solidified (F) samples.

The use of lipidic excipients like GMS supposes to consider the evalution of crystalline properties of the material on time, during processing and depending on the temperature conditions adopted during storage. Glycerides with higher melting points and stable polymorphic content are less sensitive to physical modifications and thus to the drug release rate alterations generally associated with lipidic dosage forms [16].

\subsection{Particle morphology via SEM and AFM analyses}

Surface micrographs of pure niacin and prepared niacin GMS extrudate were captured using SEM technique. The SEM micrograph of pure niacin shows large crystalline forms of drug agglomerates with ordered shape and size Fig. 3 (A). The cross sectional view at microlevel of the 
extrudates showed that niacin drug particles are dispered inside the GMS lipid matrices owing to the homogenous blend duirng the high shear melt granulation processing Fig 3 (B and C). The surface characteristics of niacin GMS extrudates showed rough disordered and crystaline structures Fig 7 (D and E), which subsequently help to dissolve drug initially when comes in contact with aqueous fluid. The extrudate systems exibit relatively rough and dense structure suggesting that the drug and hydrophobic lipid were mixed uniformly which may be the underlying reason of the sutained release drug formulations.

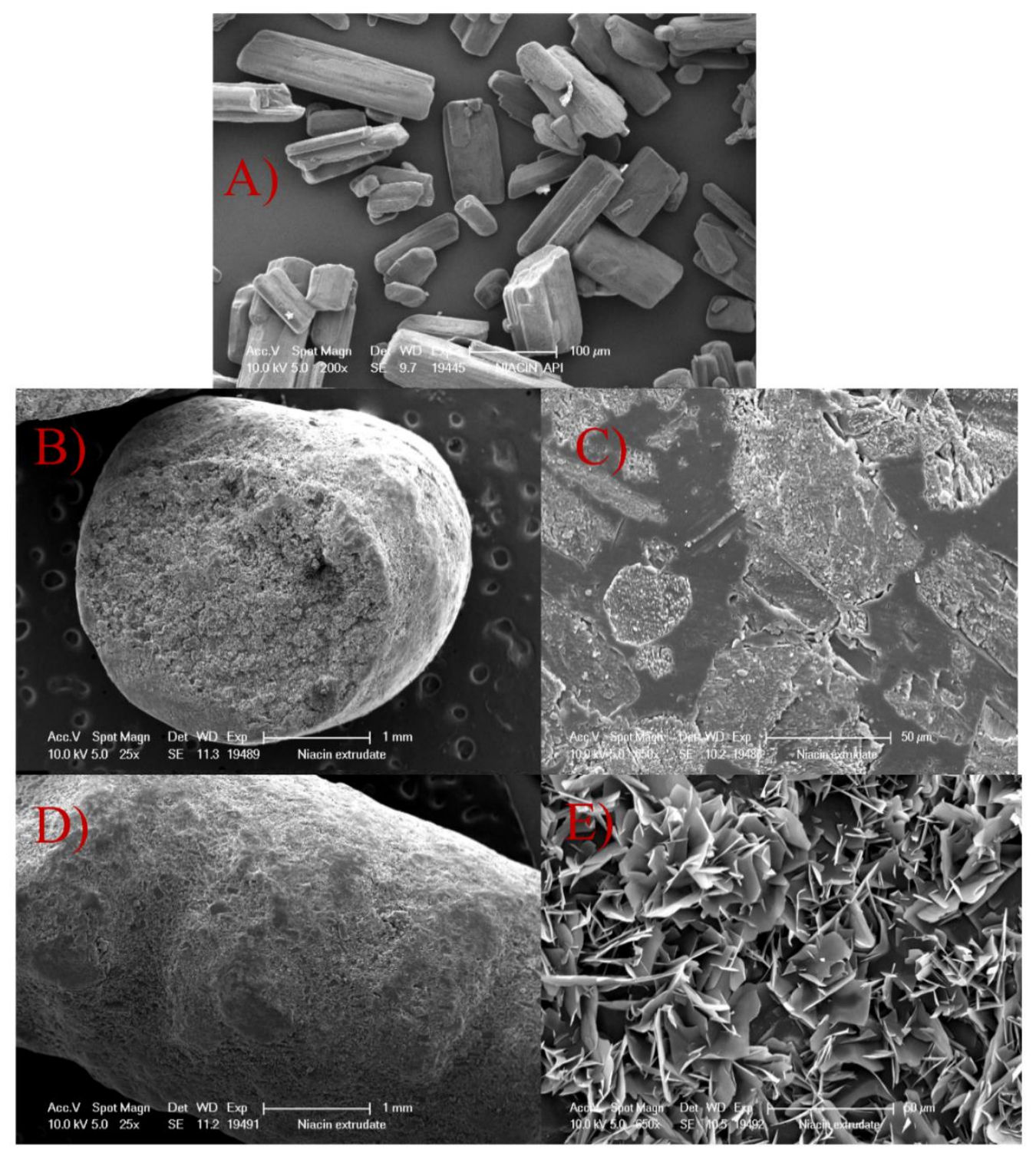

Figure 3: SEM images of A) Pure Niacin B) Cross sectional View of Niacin GMS extrudate C) Molecular View of Niacin GMS extrudate D) Logitudinal View of Niacin GMS extrudate E) 650x of Longitidinal view of Niacin GMS extrudate. 
Fractured fresh extrudate with smooth surfaces were used for microscopic investigations using AFM. Fracture surfaces were generated at determined fracture points on the outer surfaces of the extrudates. From all extrudates having the form of transparent cylindrical rods, approximately 2.5 and $3.5 \mathrm{~cm}$ in length and of $0.5 \mathrm{~cm}$ width were selected, and placed on a sheet of paper for AFM investigation. The freshly fractured extrudates were mounted on an optical glass slide by use of a 2 component epoxy resin, which hardened within 5 min. Before the hardening reaction had been completed the extrudate orientation was corrected to get the fracture surface as horizontal as possible. This step is mandatory to enable non- destructive imaging and automated sample changing within Atomic Force Microscope operations [26]. Freshly fractured extrudates on microscopic glass slides were mounted on the micrometre positioning stage of a Dimension Icon AFM. Between 10 and 25 regions per sample were programmed to be automatically characterized using the software routine "programmed move" in Tapping Mode. Height, phase, and amplitude images were collected simultaneously, using etched silicon cantilevers with a nominal spring constant of $\mathrm{k}=40-100 \mathrm{~N} / \mathrm{m}$ (JEOL AFM Probes). The typical free vibration amplitude was in the range of $\mathrm{A}=80 \mathrm{~nm}$, the images were recorded with set-point amplitudes corresponding to $60-70 \%$ of the free amplitude. Image areas of $10 \mathrm{x} 10 \mathrm{~mm}$ were recorded at a resolution of 1024 x 1024 pixels. All data were batch processed using Scanning Probe Image Processor (SPIP 5.1.1) Height data were plane-corrected by applying a 3rd order polynomial fit [27]. Molecular fracture roughness data as presented in Fig. 4, consist of cross sectional surface roughness calculated from at least 10 images on each sample. The 3D surface image of niacin GMS extrudate depicted in Fig 4, which gives morphological surface interactions in detail. The roughness parameters reflect the \% variation with respect to the topography mean height. It was concluded from the AFM analysis that there is high level of surface interaction and dense structure of drug inside lipid matrix observed in extrudates. Also the peaks and vallies observed in 3D graph that represent the crystalline drug inside the surface of extrudates. 

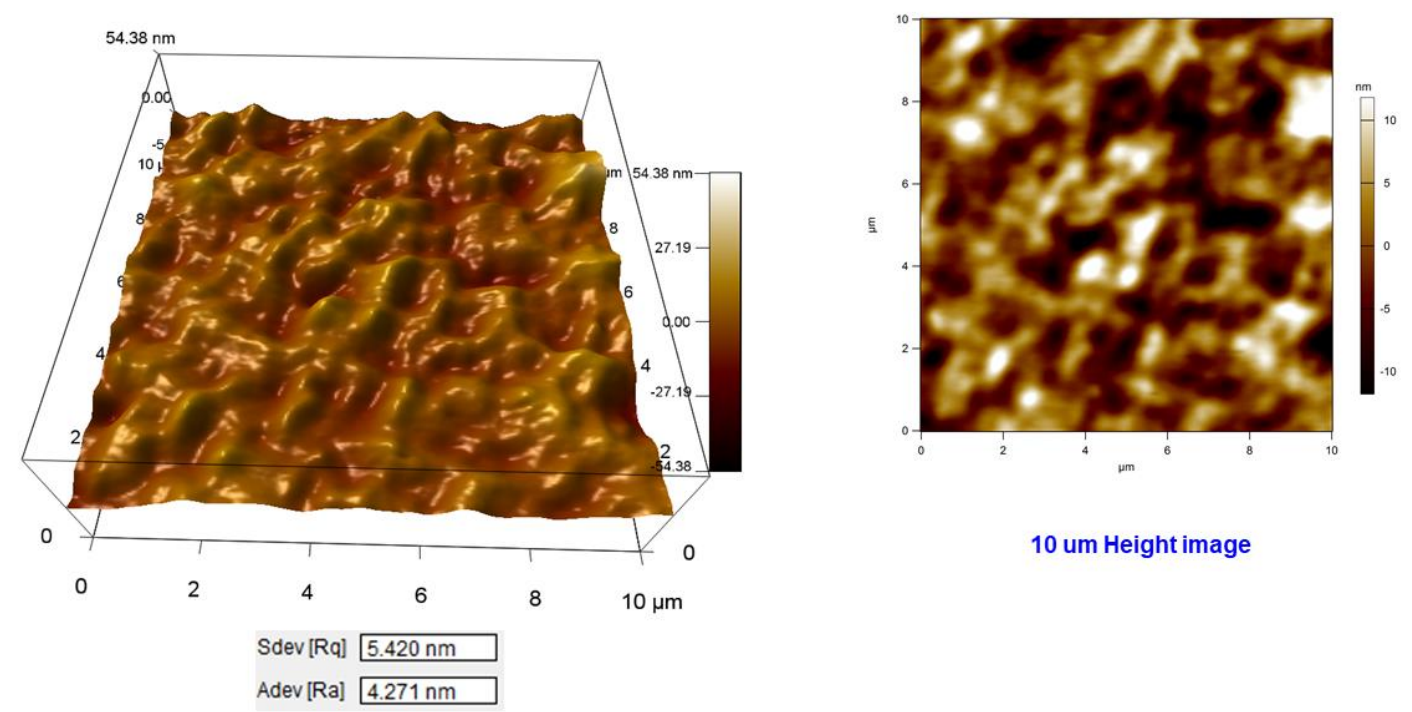

Figure 4: AFM microscopic image Niacin GMS extrudate (cross sectional).

\subsection{Melt Viscosity study of GMS}

Another object of this study is the physical evaluation of two GMS samples that have to be used as meltable binders for preparation of sustained-release tablets of niacin in high shear process, i.e melt granulation. The deformation properties of Gelol and Capmul GMS 50K (untreated) were also studied by using a temperature controlled viscometer.

The rheological evaluations of Geleol and Capmul GMS 50K determined on the solid material at different temperatures close to their melting point, have shown a pseudoplastic flow. The apparent viscosity [ point is summarised in Table 3. As seen from the table, apparent viscosity values of both GMS samples at a temperature value of $50^{\circ} \mathrm{C}$ the lipids are just started melting. The melt viscosity values are similar in range. Moreover, above this temperature (between 55 and $65{ }^{\circ} \mathrm{C}$ ), two GMS samples start behaving separately in viscosity parameters. The Geleol which tend to shift to its unstable $\alpha$ form entirely melted and thus too low viscosity values were recorded at a temperature $70^{0} \mathrm{C}$. Indeed it's the Capmul GMS 50K which provides good melt viscosity at higher temperature which is due to the presence of its stable $\beta$ form. In the temperature range $65-70^{\circ} \mathrm{C}$ it holds the structure and may provide good binding properties The comparison of melt viscosity values are represented in the graph. (Fig. 5). This ability is also depicted in DSC endotherms of Capmul GMS samples. These results show that use of stable GMS as a meltable binder presenting well distinct melting temperature could be a very interesting tool for preparation of sustained-release tablets by melt 
granulation, especially when relatively high amounts of lipidic binder have to be used to obtain appropriate drug release properties. As it was essentially discussed in a research paper [16], the careful control of the product temperature (between 45 and $50{ }^{\circ} \mathrm{C}$ ) during the pelletisation process permits to control the amount of binder melted, and thus to avoid any "overwetting" phenomenon. DSC and XRD and Melt viscosity results have shown that how two GMS samples vary in melting and performance during processing since they have different melting profiles over a wide range of temperatures, from temperature values as low as 50 to $75^{\circ} \mathrm{C}$. Moreover, results obtained by the melt viscosity studies from GMS materials show that viscosity values obtained at $65^{\circ} \mathrm{C}$ for the different lipidic mixtures are sufficient to promote the binder deformation under the action of high shearing forces developed during melt granulation process. The melt granulation technique with lipids will be an advantageous method to produce effective controlled-release tablets for high dose drugs. Therefore, the understanding of thermal and rheological characteristics of starting materials is obligatory before developing such lipidic dosage forms. As the lipidic excipients act as binders, their melting range have to be closely interconnected with the product temperature during melt granulation process in order to obtain an effective drug release while avoiding any "overwetting" phenomenon. Moreover, the rheological study of fatty binders gives a fascinating illustration of the viscosity and deformation of melting binders under high shear forces in high shear granulation $[3,28]$.

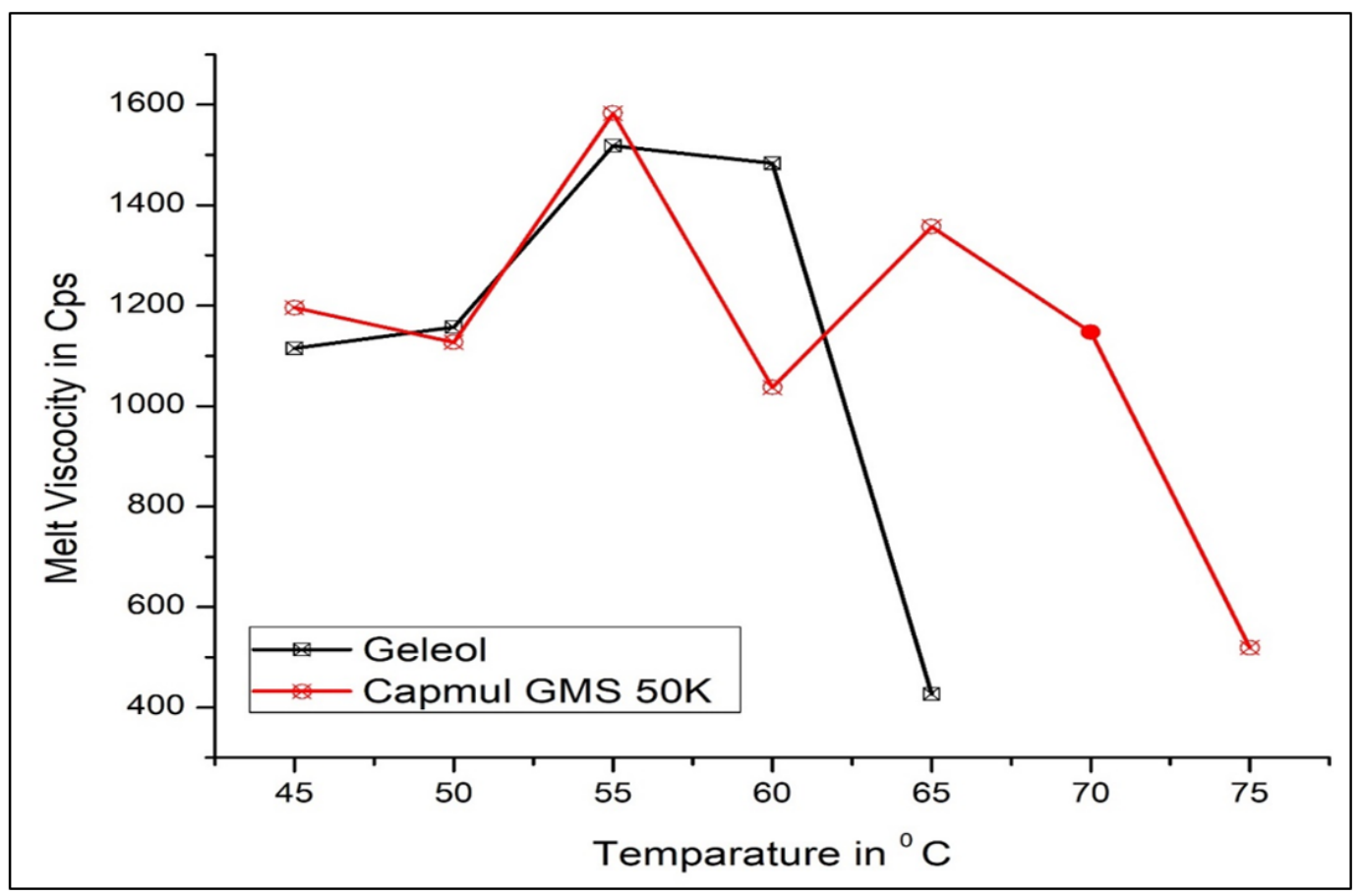


Figure 5: Melt viscosity profile of Geleol and Capmul GMS $50 \mathrm{~K}$ untreated samples.

Table 3: The apparent viscosity napp (cps) results calculated at spindle speed $50 \mathrm{rpm}$ for Geleol and Capmul GMS at different temperatures.

\begin{tabular}{|c|c|c|c|c|c|c|c|}
\hline Meltable Excipient & $\mathbf{4 5}^{\mathbf{0}} \mathbf{C}$ & $\mathbf{5 0}^{\mathbf{0}} \mathbf{C}$ & $\mathbf{5 5}^{\mathbf{0}} \mathbf{C}$ & $\mathbf{6 0}^{\mathbf{0}} \mathbf{C}$ & $\mathbf{6 5}^{\mathbf{0}} \mathbf{C}$ & $\mathbf{7 0}^{\mathbf{0}} \mathbf{C}$ & $\mathbf{7 5}^{\mathbf{0}} \mathbf{C}$ \\
\hline Geleol & 1115 & 1157 & 1518 & 1483 & 427 & $\mathrm{a}$ & a \\
\hline Capmul GMS 50K & 1196 & 1127 & 1583 & 1037 & 1357 & 1147 & 519 \\
\hline
\end{tabular}

a melted stage.

\subsection{Melt Granulation of Niacin using GMS as Matrix former}

The main disadvantage professed for lipids is their physical variability upon storage and potential influence on drug release. So far, only a few literature references endorsed the instability of drug release to polymorphic changes of lipid excipient and so considering above limitations of the lipids, we hypothesized that the GMS of two sources may impact release and stability profiles of the drug formulations. Hence we attempted to evaluate impact of ageing conditions i.e storage at $40^{\circ} \mathrm{C} / 75 \% \mathrm{RH}$ for 90 days on phase transitions of two GMS samples and to correlate these potential changes with in-vitro drug release. Niacin which is a high dose and highly water-soluble drug molecule selected as model API for developing sustained release tablets using GMS. The dose selected is $375 \mathrm{mg}$. In initial studies, the niacin along with lipid binder, GMS was evaluated in water-insoluble anti-sticking cum bulking agent i.e with microcrystalline cellulose (MCC). This blend was prepared at 70\%, 60\%, and 50\% drug loading and it processed through the extruder with all temperature control points set at $75^{\circ} \mathrm{C}$, which is above the melting temperature range of GMS. All formulations had drug content values within the range of 95-105\%. 


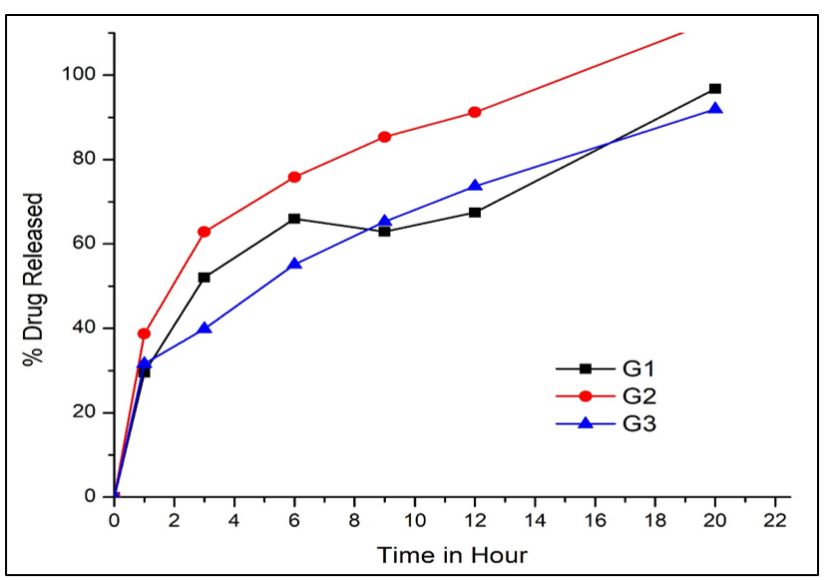

(a)

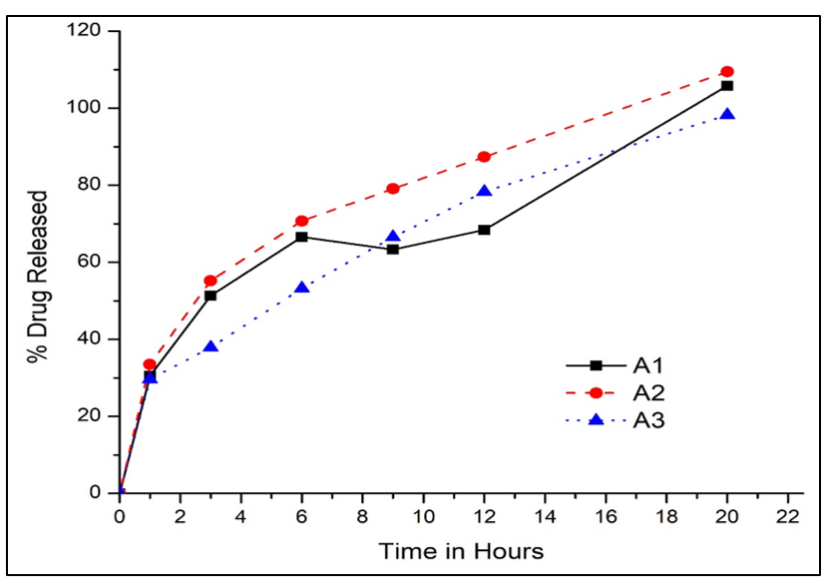

(b)

Figure 6: (a) Dissolution Profile of Niacin sustained release tablets using Geleol- Initial point; (b) Dissolution Profile of Niacin sustained release tablets using Capmul GMS- Initial point.

The melting point and stability of the meltable lipid binder are not only critical for process control of melt granulation process, but likely plays a critical role in controlling the drug release rate, which is discussed in later part of article. Relatively little work has been commenced so far to reveal structural properties of GMS as a function of its thermal behavior. Like the other lipid excipients, GMS is manufactured using vegetable source materials which present a complex chemical composition. The ability of each glyceridic compound to present different polymorphic form adds another source of complexity. Also, the exact composition of mono, di, triacylglycerols in GMS is maintained differently by each company. But these GMS samples would pass the pharmacopeial requirements of monographs. When it comes to the performance even small variation in fatty acid composition and more importantly the polymorphic form of lipid plays a huge role in drug delivery system.

Tablet dissolution study provides evidence that the GMS was completely melted and it has coated the niacin. Melt granulation process appear to have initial around, 30\%, burst effect. The milling process resulted in particles having a rough surface morphology, which exposes niacin to dissolution media. Though there is an initial burst effect which is also within the acceptance criteria, the twin screw granules are better encapsulated by portion of GMS that melted during the process. Following the burst release from tablets, an additional $70 \%$ of the drug was released over next 18 h. (Fig 6 a-b ). A probable justification, held in the evidence that all of GMS is melted during process, which has coated the particles of niacin. This geometry helps to control the release 
profile of drug in dissolution medium. $(10,25)$

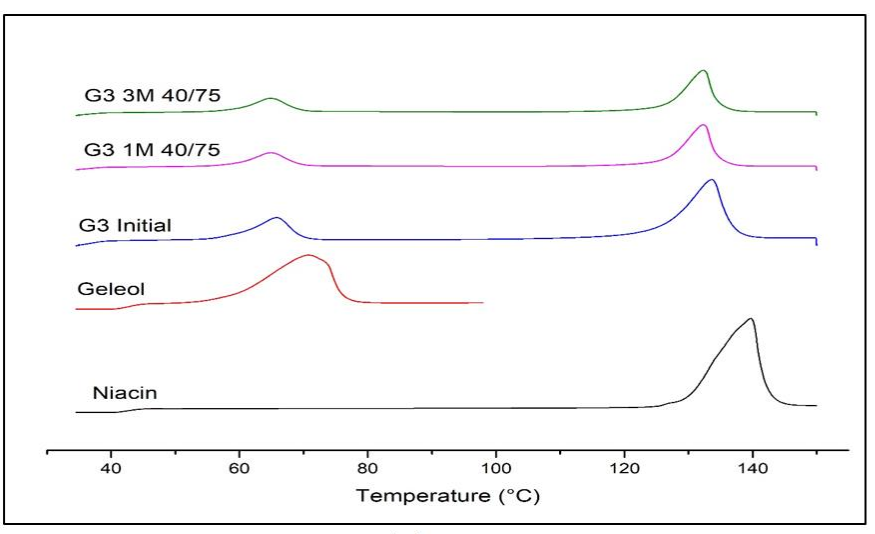

(a)

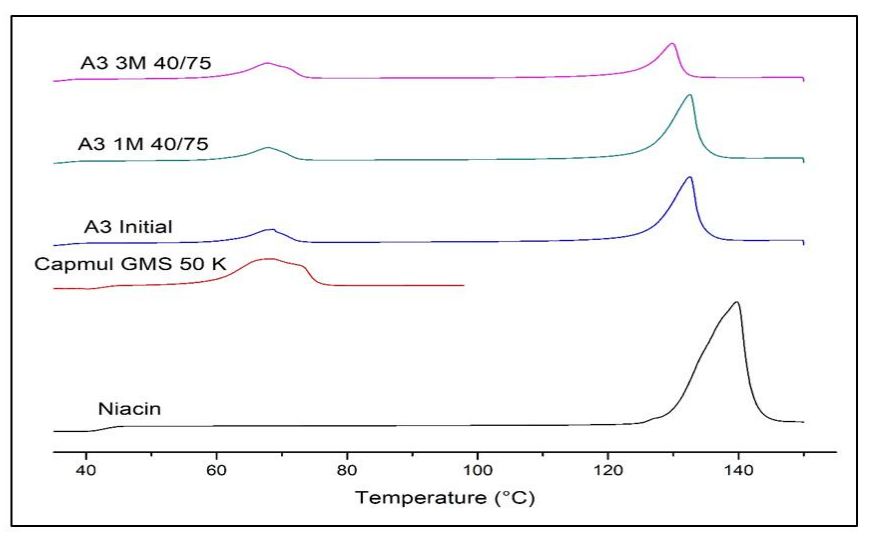

(b)

Figure 7: (a) DSC thermograms -Influence of storage conditions on stability of Tablets- Lipid "Geleol”; (b) DSC thermograms- Influence of storage conditions on stability of Tablets- Lipid "Capmul GMS 50K”.

It was reported that polymorphic changes in lipid-based dosage forms are known to modify drug release and such occurrences were critical to understanding early pharmaceutical dosage forms, such as suppositories and solid lipid matrices [29, 30]. Initial analytical experiments were completed to ensure that the DSC and XRD methodologies were capable of detecting the various polymorphs of GMS samples. Two GMS samples were evaluated by fusion experiments, which confirmed the ability of the DSC methodology to detect the polymorph. Geleol has a peak endotherm at $69.25^{\circ} \mathrm{C}$ which started melting from $56.83^{\circ} \mathrm{C}$ to $75.07^{\circ} \mathrm{C}$. Also the niacin has major melting endotherm at $137.82^{\circ} \mathrm{C}$. In the initial niacin with Geleol tablet samples the peak endotherm of niacin and geleol was slightly shifted on lower end i.e at $65.84^{\circ} \mathrm{C}$ and $133.6^{\circ} \mathrm{C}$ (Fig. 7a). In the accelerated stability conditions i.e $40^{\circ} \mathrm{C} / 75 \% \mathrm{RH}$ for 90 days the endotherms of both niacin and geleol was slightly shifted. This observation was correlated with DSC endotherms of heat treated and aged samples (keeping at $50^{\circ} \mathrm{C}$ for 48 hours). There also the shift of endotherms of geleol was identified. Similarly, the Capmul GMS $50 \mathrm{~K}$ has endotherm at $67.36^{\circ}$. The tablets prepared by using capmul GMS $50 \mathrm{~K}$ showed similar endotherms at same positions. During the stability studies, the endotherms were found at same positions (Fig. 7b).This observation is also correlated with the heat treated and aged samples. The melting endotherm for capmul GMS 50K was found at the same position means a stable polymorphic form of GMS is present in tablets. To recertify 
this observation X-ray diffraction helped to identify the structural stability of Capmul GMS $50 \mathrm{~K}$ samples. The two major diffraction peaks of $19.53^{\circ} \& 23.18^{0}$ were observed in lower intensities at similar positions in stability samples. (Fig. 8a). Both DSC and X-ray Diffraction techniques confirmed the crystalline form of niacin which is stable during the stability study. The stable $\beta$ form in Capmul GMS also confirmed through XRD. This finding is correlated with findigs of Haupt et al. where he has reported the transformation from an unstable $\alpha$-form into the energetically stable $\beta$-form was important is maintaining the stability of lipid based dosage form. A benefit of this tempering step is the nonexistence of polymorphic transformations during storage and therefore, missing changes in drug release [31, 32].

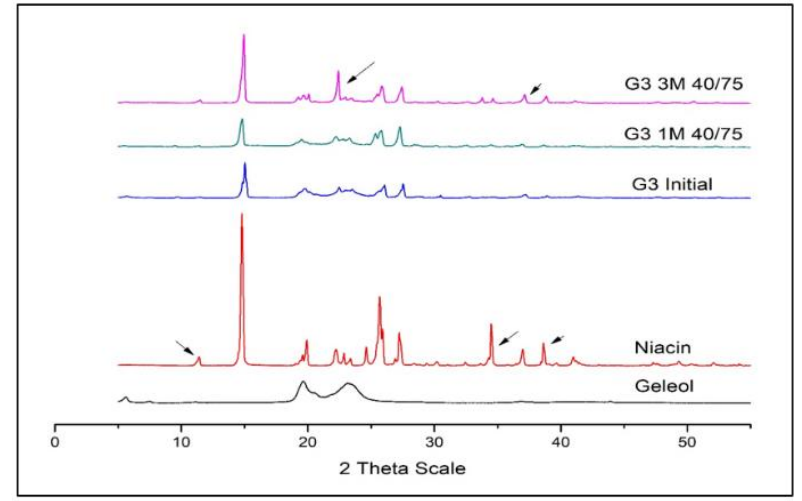

(a)

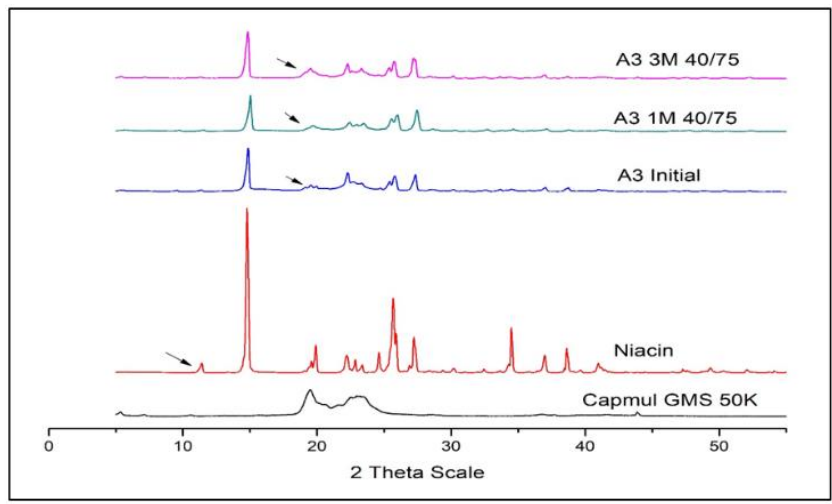

(b)

Figure 8: (a) X-ray Diffractograms-Influence of storage conditions on stability of Tablets- Lipid "Geleol”; (b) X-ray Diffractograms -Influence of storage conditions on stability of Tablets- Lipid "Capmul GMS 50K”.

Opposite to this observations while reviewing X-ray Diffraction patterns of Geleol samples during the stability study after 3 months there was a single peak observed at $23.24^{0}$. which is of $\alpha$ form of GMS. This $\alpha$ form was also observed and identified by T. Yajima et.al (2002). The least stable polymorphic form, $\alpha$ is formed while at very slow cooling rates and this present as amorphous form outside the matrix. While the the stable $\beta$ form is a crystalline form and is scattered in dense structure $[11,23,33]$. This change in stable $\beta$ form to an unstable $\alpha$ form affected the dissolution profile at 3 months stability time points (Fig $\mathbf{8 b}$ ). It is important to note here that crystalline highly water-soluble niacin was started releasing early from the unstable Geleol coat $[34,35]$. When we compared the dissolution profiles of niacin tablets prepared by using Capmul GMS $50 \mathrm{~K}$, there are no any major changes into it. (Fig. 9). For instance, the transformation from 
the thermodynamically instable and less dense $\alpha$-form to the most stable and densely packed $\beta$ form had resulted in the reduction of wettability [36-38]. This is the reason of usage of stable $\beta$ form, in sustained release dosage forms.

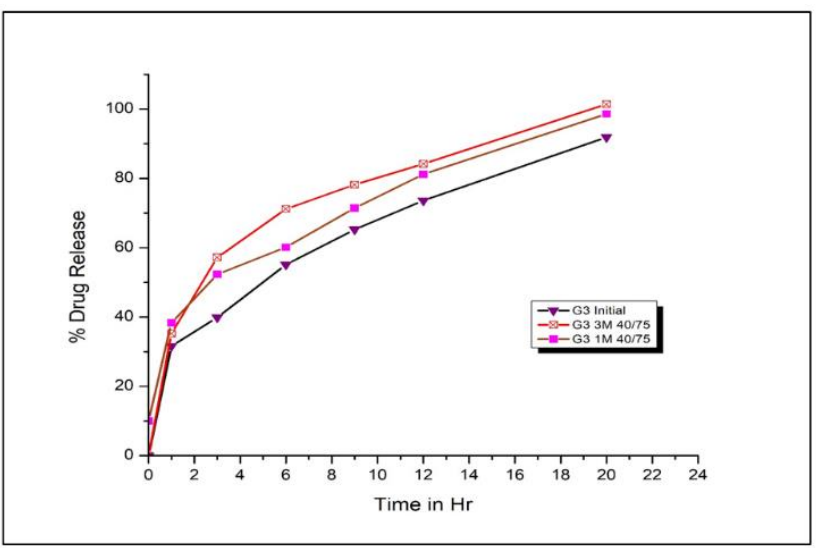

(a)

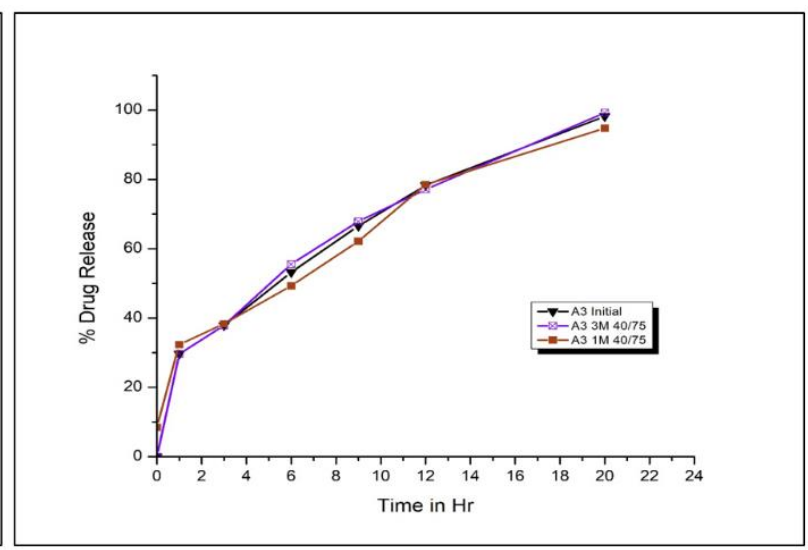

(b)

Figure 9: (a) Influence of stability condition on Dissolution of tablets-Lipid "Geleol"; (b) Influence of stability condition on Dissolution of tablets-Lipid "Capmul GMS 50K".

\section{Conclusions:}

The chemical composition of glycerides used for the manufacturing of solid lipid extrudates was found to have a large influence on solid-state behavior and dissolution profiles. This study has underlined the significance of considering the physical, thermal and rheological characteristics of GMS in order to superior control the properties of sustained-release formulations. The chemical and physical complexity of lipophilic binders exhibits quite a complex behaviour, i.e. melting and crystallisation, polymorphism. Two untreated and freshly solidified Geleol and Capmul GMS $50 \mathrm{~K}$ samples present different crystal state which was investigated by different analytical techniques. The rate of crystallisation was found to be more rapid for geleol and is highly dependent on ageing and heat treatment process. As a polymorphic form change was detected during melt granulations process it is very important to select the right starting material for drug delivery system. Due to there difference in fatty acid composition the properties the GMS influence release of the drug. In a detailed study of both GMS lipids, the Geleol led to increased incidence of the unstable $\alpha$-form of the GMS leading to recrystallization of stable $\beta$ form over time which causes fractal structures on the extrudate surface deteriorating dissolution properties. While the stable $\beta$ form withstands the heat treatment during melt granulation process. Storage experiments 
under accelerated and ambient conditions shown a strong influence of temperature on the recrystallization kinetics. The outcomes of this study help to interpret the complex solid-state behaviour of solid lipid extrudates with different compositions which simplify the development of suitable lipid-based oral dosage forms.

Conflicts of Interest: The authors declare no conflict of interest.

\section{References}

1. Maniruzzaman M, Islam MT, Halsey S, Amin D, Douroumis D. Novel Controlled Release Polymer-Lipid Formulations Processed by Hot Melt Extrusion. AAPS PharmSciTech. 2016, 17(1), 191-199. doi: 10.1208/s12249-015-0470-2

2. Patil H, Tiwari R V., Repka MA. Hot-Melt Extrusion: from Theory to Application in Pharmaceutical Formulation. AAPS PharmSciTech. 2015, 17(1), 20-42. doi: 10.1208/s12249-015-0360-7

3. Jannin V, Rodier JD, Musakhanian J. Polyoxylglycerides and glycerides: Effects of manufacturing parameters on API stability, excipient functionality and processing. Int $\mathrm{J}$ Pharm. 2014, 466(1-2), 109-121. doi: 10.1016/j.ijpharm.2014.03.007

4. Aburahma MH, Badr-Eldin SM. Compritol 888 ATO: a multifunctional lipid excipient in drug delivery systems and nanopharmaceuticals. Expert Opin Drug Deliv. 2014, 11(12), 1865-1883. doi: 10.1517/17425247.2014.935335

5. Keen JM, Foley CJ, Hughey JR, Bennett RC, Jannin V, Rosiaux Y, et al. Continuous twin screw melt granulation of glyceryl behenate: Development of controlled release tramadol hydrochloride tablets for improved safety. Int J Pharm. 2015, 487(1-2), 72-80. doi: 10.1016/j.ijpharm.2015.03.058

6. Knezevic Z, Gosak D, Hraste M, Rausl D, Khan MZI. Application of hot-melt coating process for designing a lipid based controlled release drug delivery system for highly aqueous soluble drugs. Chem Pharm Bull. 2009, 57(5), 464-471.

7. Keen JM, Hughey JR, Bennett RC, Jannin V, Rosiaux Y, Marchaud D, et al. Effect of tablet structure on controlled release from supersaturating solid dispersions containing glyceryl behenate. Mol Pharm. 2015, 12(1), 120-126. doi: 10.1021/mp500480y

8. Rosiaux, Y., Jannin, V., Hughes, S., Marchaud, D., Solid lipid excipients as matrix agents for sustained drug delivery. J. Control. Release. 2014, 188, 18-30. doi:10.1007/978-3-319-20206-8_9

9. Barakat NS, Elbagory IM, Almurshedi AS. Formulation, release characteristics and bioavailability study of oral monolithic matrix tablets containing carbamazepine. AAPS PharmSciTech. 2008, 9(3), 931-938. doi: 10.1208/s12249-008-9108-y

10. Aoki H, Iwao Y, Mizoguchi M, Noguchi S, Itai S. Clarithromycin highly-loaded gastrofloating fine granules prepared by high-shear melt granulation can enhance the efficacy of Helicobacter pylori eradication. Eur J Pharm Biopharm. 2015, 92, 22-27. doi: 10.1016/j.ejpb.2015.02.012

11. Howland H, Fahmy R, Hoag SW. Analysis of curing of a sustained release coating formulation by application of NIR spectroscopy to monitor changes associated with glyceryl monostearate. Drug Dev Ind Pharm. 2014, 9045, 1-11. doi: $10.3109 / 03639045.2014 .947505$ 
12. Vithani K, Maniruzzaman M, Slipper IJ, Mostafa S, Miolane C, Cuppok Y, et al. Sustained release solid lipid matrices processed by hot-melt extrusion (HME). Colloids Surfaces B Biointerfaces. 2013, 110, 403-410. doi: 10.1016/j.colsurfb.2013.03.060

13. Priyanka K, Abdul Hasan SA. Preparation and Evaluation of Montelukast Sodium Loaded Solid Lipid Nanoparticles. J Young Pharm. 2012, 4(3), 129-137. doi: 10.4103/09751483.100016.

14. Pivette P, Faivre V, Brubach JB, Daste G, Ollivon M, Lesieur S. Polymorphism of glyceryl behenates: From the individual compounds to the pharmaceutical excipient. Chem Phys Lipids. 2014, 183, 191-203. doi: 10.1016/j.chemphyslip.2014.07.005

15. Sato K. Crystallization behaviour of fats and lipids - a review. Chem Eng Sci. 2001, 56(7), 2255-2265. doi.org/10.1016/S0009-2509(00)00458-9

16. Hamdani J, Moës AJ, Amighi K. Physical and thermal characterisation of Precirol® and Compritol ${ }^{\circledR}$ as lipophilic glycerides used for the preparation of controlled-release matrix pellets. Int J Pharm. 2003, 260(1), 47-57. doi.org/10.1016/S0378-5173(03)00229-1

17. Lauer M, Siam M, Tardio J, et al. Rapid assessment of homogeneity and stability of amorphous solid dispersions by atomic force microscopy-from bench to batch. Pharm Res 2013, 30, 2010-2022. doi: 10.1007/s11095-013-1045-0

18. Taylor AK Glyceryl Monosterate. In Handbook of Pharmaceutical Excipients Edition-6; Rowe RC, Sheskey PJ, Quinn ME Pharmaceutical Press London UK. 2009; 290-293.

19. Ensio L, Pirjo APK. On the structural behaviour of triglycerides with time. Int J Pharm. 1988, 43, 241-247. doi.org/10.1016/0378-5173(88)90280-3

20. Cornish R. Studies of Glyceryl Monostearate. J Soc Cosmet Chem. 1968, 117(19), 109117.

21. Yajima T, Itai S, Takeuchi H, Kawashima Y. Determination of Optimum Processing Temperature for Transformation of Glyceryl Monostearate. Chem Pharm Bull. 2002, 50(11), 1430-1433. doi.org/10.1248/cpb.50.1430

22. Xia D, Cui F, Gan Y, Mu H, Yang M. Design of Lipid Matrix Particles for Fenofibrate: Effect of Polymorphism of Glycerol Monostearate on Drug Incorporation. J Pharm Sci. 2014, 103, 697-705. doi: 10.1002/jps.23830.

23. Jingying L, C CP, Mingshi Y, M NH, Huiling M. The impact of particle preparation methods and polymorphic stability of lipid excipients on protein distribution in microparticles. Drug Dev Ind Pharm. 2017, doi.org/10.1080/03639045.2017.1361967

24. Lamm, M.S., DiNunzio, J., Khawaja, N.N., Crocker, L.S., Pecora, A. Assessing Mixing Quality of a Copovidone-TPGS Hot Melt Extrusion Process with Atomic Force Microscopy and Differential Scanning Calorimetry. AAPS PharmSciTech. 2016, 17, 8998. doi:10.1208/s12249-015-0387-9.

25. Almeida A, Saerens L, De Beer T, et al. Upscaling and in-line process monitoring via spectroscopic techniques of ethylene vinyl acetate hot-melt extruded formulations. Int $\mathbf{J}$ Pharm 2012, 439, 223-229. doi.org/10.1016/j.ijpharm.2012.09.037

26. Balasubramaniam VM, Maleky Farnaz MZ. Thermal E ff ects on Lipids Crystallization Kinetics under High Pressure. Cryst Growth Des. 2017, 17, 4835-4843. doi 10.1021/acs.cgd.7b00768

27. Jannin V, Cuppok Y. Hot-melt coating with lipid excipients. Int J Pharm.2013, 457(2), 480-487. doi: 10.1016/j.ijpharm.2012.10.026

28. Aoki H, Iwao Y, Uchimoto T, Noguchi S, Kajihara R, Takahashi K, et al. Fine granules showing sustained drug release prepared by high-shear melt granulation using triglycerin 
full behenate and milled microcrystalline cellulose. Int J Pharm. 2015, 478(2), 530-539. doi: 10.1016/j.ijpharm.2014.11.058

29. Pattarino F, Bettini R, Foglio A, Della A. Polymorphism and kinetic behavior of binary mixtures of triglycerides. Int $\mathrm{J}$ Pharm. 2014, 473(1-2), 87-94. doi: 10.1016/j.ijpharm.2014.06.042

30. Windbergs M, Strachan CJ, Kleinebudde P. Influence of the composition of glycerides on the solid-state behaviour and the dissolution profiles of solid lipid extrudates. Int J Pharm. 2009, 381(2), 184-191. doi: 10.1016/j.ijpharm.2009.03.002

31. Haupt M, Thommes M, Heidenreich A, Breitkreutz J. Lipid-based intravesical drug delivery systems with controlled release of trospium chloride for the urinary bladder. $\mathbf{J}$ Control Release. 2013, 170(2), 161-166. doi: 10.1016/j.jconrel.2013.05.018.

32. Rosiaux Y. New insights into the stability of SR lipid matrix tablets. In: AAPS Annual Meeting and Exposition, Orange County Convention Center, Orlando, Florida USA Oct 25-29 2015. p. 70070.

33. Sato K, García LB, Calvet T, Àngel M, Diarte C. External factors affecting polymorphic crystallization of lipids. Eur J Lipid Sci Technol. 2013, 115, 1224-1238. doi.org/10.1002/ejlt.201300049

34. Cullis. P.R KB DE. Lipid Polymorphism and the Functional Roles of Lipids In. Biochim Biophys Acta. 1979, 559, 399-420. doi.org/10.1016/0304-4157(79)90012-1

35. Alfutimie A, Al-janabi N, Curtis R, Tiddy GJT. Physicochemical and Engineering Aspects The Effect of monoglycerides on the crystallisation of triglyceride. Colloids Surfaces A Physicochem Eng Asp. 2016, 494, 170-179. DOI: 10.1016/j.colsurfa.2016.01.029

36. Becker K, Salar-behzadi S, Zimmer A. Solvent-Free Melting Techniques for the Preparation of Lipid-Based Solid Oral Formulations. Pharm Res. 2015, 32, 1519-1545. doi: 10.1007/s11095-015-1661-y

37. Wang FC, Marangoni AG. Effect of Intrinsic and Extrinsic Factors on the Stability of the $\alpha$-gel Phase of a Glyceryl Monostearate-Water System. RSC Adv. 2015, 5, 43121-43129. DOI 10.1039/C5RA05045F

38. Paula A, Ribeiro B, Masuchi MH, Miyasaki EK. Crystallization modifiers in lipid systems. J Food Sci Technol. 2015;52, 3925-3946. doi: 10.1007/s13197-014-1587-0 